\title{
Über das Verhalten einiger Pilze zu organischen Säuren.
}

I. Mitteilung.

Von

R. O. Herzog und O. Ripke.

(Aus dem chemischen Institut der Technischen Hochschule, Karlsruhe.) ${ }^{1}$ )

(Der Redaktion zugegangen am 22. Juni 1911.)

Die folgenden Mitteilungen betreffen Versuche, die im Anschluß an die von R. O. Herzog und A. Meier ${ }^{2}$ ) ausgeführten vorgenommen wurden. Es hat sich ergeben, daß zum Teil unerwartete Verhältnisse eintraten, deren Besprechung aber wohl zweckmäßig im Zusammenhang mit den Versuchen erfolgt.

1. Das Wachstum einiger Pilze in saurer Lösung und das Verschwinden der Säuren.

Mycoderma cerevisae, Monilia candida und Oidium lactis wurden auf Nährlösungen übergeimpft, die $1 / 2-3 \%$ der im folgenden genannten Säuren enthielten. Als Nährlösung wurde die bekannte Mischung nach Uschinsky ${ }^{3}$ ) angewandt. Im folgenden ist angegeben, wieviel Prozente der zugesetzten Säuren nach 11/2 Monaten von den Pilzen zum Verschwinden gebracht wurden (s. Tab.).

1) Die letzten Versuche sind von dem Einen von uns (R.) im Physiolog. Institut der Universität Heidelberg ausgeführt worden. Herrn Geheimrat Kossel sei für die liebenswürdige Erlaubnis dazu der verbindlichste Dank ausgesprochen!

2) Diese Zeitschrift, Bd. 57, S. 35 (1908), Bd. 59, S. 57 (1909).

3) Uschinskys Nährlösung enthält: $30-40 \mathrm{~g}$ Glycerin, 5-7 g Kochsalz, 0,1 g Chlorcalcium, 0,2-0,4 Magnesiumsulfat, 2,5-3 g Kaliumphosphat, 6-7 g Ammoniumlactat, 3-4 g Asparagin gelöst in einem Liter Leitungswasser. 
Über das Verhalten einiger Pilze zu organischen Säuren. I. 285

\begin{tabular}{|c|c|c|c|}
\hline Säure & Pilz & $\begin{array}{l}\text { Säuregehalt } \\
\text { der ursprüngl. } \\
\text { Lösung } \\
\text { o/o }\end{array}$ & $\begin{array}{l}\text { Hiervon sind } \\
\qquad \% \\
\text { verschwunden }\end{array}$ \\
\hline Ameisensäure & Mon. cand. & 0,33 & 77 \\
\hline Essigsäure & Myc. cerev. & 0,75 & 88 \\
\hline$>$ & 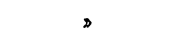 & 1,12 & 89 \\
\hline Propionsäure & 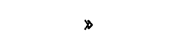 & 0,73 & 99 \\
\hline$>$ & $>$ & 1,09 & 28 \\
\hline Bernsteinsäure & , & 0,67 & 82 \\
\hline , & $»$ & 1,18 & 88 \\
\hline$\triangleright$ & $\gg$ & 2,24 & 94 \\
\hline$\triangleright$ & Oid. lact. & 0,67 & 75 \\
\hline$\triangleright$ & $>$ & 1,18 & 80 \\
\hline$\triangleright$ & s & 2,24 & 89 \\
\hline Milchsäure & Myc. cerev. & 0,86 & 76 \\
\hline$>$ & $>$ & 1,98 & 91 \\
\hline$D$ & , & 3,13 & 94 \\
\hline$>$ & Oid. lact. & 0,86 & 68 \\
\hline , & $\gg$ & 1,98 & 78 \\
\hline$>$ & , & 3,13 & 85 \\
\hline Äpfelsäure & Myc. cerev. & 0,84 & 84 \\
\hline , & 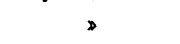 & 1,34 & 90 \\
\hline$\triangleright$ & s & 3,18 & 95 \\
\hline$\triangleright$ & Mon. cand. & 0,84 & 86 \\
\hline 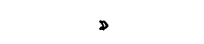 & , & 1,34 & 79 \\
\hline , & Oid. lact. & 1,34 & 83 \\
\hline Glykollsäure & Myc. cerev. & 0,95 & 80 \\
\hline , & $>$ & 1,34 & 86 \\
\hline$>$ & Mon. cand. & 0,95 & 55 \\
\hline$>$ & Oid. lact. & 0,95 & 85 \\
\hline Traubensäure & Myc. cerev. & 0,86 & 48 \\
\hline$>$ & , & 1,19 & 29 \\
\hline$>$ & Mon. cand. & 0,86 & 50 \\
\hline 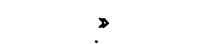 & Oid. lact. & 0,86 & 35 \\
\hline Citronensäure & Myc. cerev. & 0,77 & 46 \\
\hline , & Mon. cand. & 0,77 & 82 \\
\hline
\end{tabular}

Die Versuche zeigen sowohl die spezifische Elektionsfähigkeit wie die mitunter erhebliche Säurefestigkeit der Pilze. 
2. Das Verhalten von abgetötetem Oidium lactis gegenüber Säuren.

Im folgenden sind Versuche mitgeteilt, die in ähnlicher Weise durchgeführt sind, wie in der Arbeit von Herzog und A. Meier (l. c.) beschrieben wurde. Auf Bierwürze gutgewachsene Pilze wurden mit Aceton und Äther getötet und getrocknet, mit verdünnter Säurelösung und im Parallelversuch mit destilliertem Wasser überschichtet - unter Zusatz von Toluol als Antisepticum - und ein Luftstrom durch die Gefäße geschickt. Die vom letzteren mitgeführte Kohlensäure wurde bestimmt. Nach Ablauf des Versuches wurde auch die freie Säure titriert. Da das Pilzmycel von der Nährlösung her und vielleicht auch durch Autolyse sauer reagierte, wurde zum Vergleich auch die Lösung des Kolbens titriert, in welchem Wasser zu der Pilzmasse zugesetzt worden war.

Versuch 1.

Kölbchen a enthielt $100 \mathrm{ccm}$ Wasser (dest.), 30 Tropfen Toluol, ca. $10 \mathrm{~g}$ Pilzsubstanz.

Kölbchen b enthielt dasselbe wie a, außerdem noch $1,143 \mathrm{~g}$ Milchsäure.

Kohlensäureproduktion bei :

\begin{tabular}{|c|c|c|c|}
\hline \multirow{3}{*}{\multicolumn{2}{|c|}{$\begin{array}{c}\text { nach } 18 \text { Stunden } \\
» 24\end{array}$}} & \multirow{3}{*}{$\begin{array}{l}\text { a) } \\
0,0006 \\
0,000\end{array}$} & \multirow{2}{*}{$\begin{array}{c}\text { b) } \\
0,0030\end{array}$} \\
\hline & & & \\
\hline & & & 0,000 \\
\hline nach & 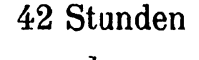 & 0,0006 & 0,0030 \\
\hline
\end{tabular}

$10 \mathrm{ccm}$ der ursprünglich angewandten Milchsäurelösung verbrauchten vor dem Versuch $12,7 \mathrm{ccm} 0,1-\mathrm{n}-\mathrm{NaOH}$.

$10 \mathrm{ccm}$ der Flüssigkeit in a (Wasser) verbrauchten nach dem Versuch 3,9 ccm 0,1-n- $\mathrm{NaOH}$.

$10 \mathrm{ccm}$ der Flüssigkeit in $\mathrm{b}$ (Milchsäure) verbrauchten nach dem Versuch $14,5 \mathrm{ccm} 0,1-\mathrm{n}-\mathrm{NaOH}$.

\section{Versuch 2.}

Kölbchen a enthielt $50 \mathrm{ccm}$ Wasser (dest.) 20 Tropfen Toluol, 5,6 g Pilzsubstanz. Milchsäure.

Kölbchen $\mathrm{b}$ enthielt dasselbe wie $\mathrm{a}+0,652 \mathrm{~g}$ freie 
Über das Verhalten einiger Pilze zu organischen Säuren. I. 287

Kölbchen c enthielt dasselbe wie a $+0,6 \mathrm{~g}$ milchsaures Natrium.

Kohlensäureproduktion bei:

a)

nach 20 Stunden

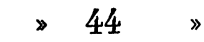

nach 64 Stunden
0,0050

b)

\begin{tabular}{lll}
0,000 & 0,0209 & 0,000 \\
\hline 0,0050 & 0,0529 & $0,0098 \mathrm{~g} \mathrm{CO}_{2}$.
\end{tabular}

$10 \mathrm{ccm}$ der ursprünglich angewandten Milchsäurelösung verbrauchten $14,5 \mathrm{ccm} 0,1-\mathrm{n}-\mathrm{NaOH}$.

$10 \mathrm{ccm}$ der Flüssigkeit in a (Wasser) verbrauchten nach dem Versuch $1,8 \mathrm{ccm}$ 0,1-n- $\mathrm{NaOH}$.

$10 \mathrm{ccm}$ der Flüssigkeit in $\mathrm{b}$ (Milchsäure) verbrauchten nach dem Versuch 13,0 ccm 0,1-n-NaOH.

Die Kohlensäureproduktion ist in beiden Versuchen bei Zusatz von Milchsäure erheblich größer als ohne solche, Zusatz von milchsaurem Natrium wirkt nicht so günstig. Die Acidität nimmt ab, wenn man die Abgabe von Säure an die Lösung (Vergleich: Wasser) in Rechnung zieht.

Negative Resultate wurden erhalten, wenn statt Milchsäure Bernstein- oder Mandelsäure angewandt wurde, obwohl die lebenden Pilze, wenigstens die erstere Säure sicher zum Verschwinden brachten (vgl. obige Tabelle).

Flasche a enthielt $150 \mathrm{ccm}$ destilliertes Wasser, 60 Tropfen Toluol und $11,7 \mathrm{~g}$ Pilzsubstanz.

Flasche $b$ enthielt dasselbe wie $a+1,4820 \mathrm{~g}$ Bernsteinsäure.

Flasche $\mathrm{c}$ enthielt $0,6916 \mathrm{~g}$ bernsteinsaures Natrium, sonst dasselbe wie a.

Kohlensäureproduktion bei :

a)

nach 14 Stunden

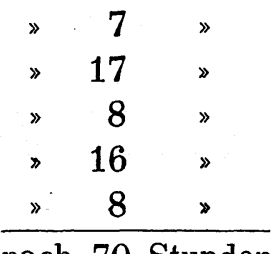

nach 70 Stunden

\begin{tabular}{lll}
0,0192 & 0,0076 & 0,0098 \\
0,0070 & 0,0036 & 0,0014 \\
0,0070 & 0,0082 & 0,0020 \\
0,0178 & 0,0026 & 0,0014 \\
0,0320 & 0,0052 & 0,0026 \\
0,0016 & 0,0022 & 0,0012 \\
\hline 0,0846 & 0,0294 & 0,0184 g
\end{tabular}


$10 \mathrm{ccm}$ der ursprünglich angewandten Bernsteinsäure verbrauchten $16,75 \mathrm{ccm} 0,1-\mathrm{n}-\mathrm{NaOH}$.

$10 \mathrm{ccm}$ der Flüssigkeit in a (Wasser) verbrauchten nach dem Versuch $0,8 \mathrm{ccm} 0,1-\mathrm{n}-\mathrm{NaOH}$.

$10 \mathrm{ccm}$ Flüssigkeit in $\mathrm{b}$ (Bernsteinsäure) verbrauchten nach dem Versuch $17,9 \mathrm{ccm} 0,1-\mathrm{n}-\mathrm{NaOH}$.

$10 \mathrm{ccm}$ Flüssigkeit in c (bernsteinsaures Natrium) verbrauchten nach dem Versuch $1,55 \mathrm{ccm} 0,1-\mathrm{n}-\mathrm{NaOH}$.

Dasselbe negative Ergebnis brachte ein zweiter Versuch mit Bernsteinsäure $(2,61 \mathrm{~g}$ auf $150 \mathrm{ccm}$ Wasser $)$ und einer mit Mandelsäure $(1,527 \mathrm{~g}$ freie Mandelsäure, in einem Parallelver-

- such 1,0486 g mandelsaures Natrium auf je $150 \mathrm{ccm}$ Wasser).

Der folgende Versuch ist wieder mit Milchsäure angestellt, nur wurde das (gut abgepreßte) Pilzmycel nicht mit Aceton getötet; sondern in flüssige Luft eingetragen und in derselben bis $\mathrm{zu}$ ihrer vollständigen Verdampfung stundenlang gehalten.

Kölbchen a enthielt $50 \mathrm{ccm}$ destilliertes Wasser, $1 / 2 \%$ Fluornatrium oder Antisepticum und ca. $9 \mathrm{~g}$ der Pilzsubstanz. Kölbchen b enthielt dasselbe wie $a+0,360$ g Milchsäure. Kohlensäureproduktion bei:

\begin{tabular}{|c|c|c|}
\hline 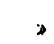 & 10 & $\triangleright$ \\
\hline 》 & 12 & » \\
\hline 》 & 10 & $\nu$ \\
\hline 》 & 25 & » \\
\hline » & 14 & » \\
\hline
\end{tabular}

a)

\begin{tabular}{ll}
0,0042 & 0,0234 \\
0,000 & 0,0040 \\
0,000 & 0,0024 \\
0,0024 & 0,0024 \\
0,0092 & 0,0074 \\
0,0068 & 0,0032 \\
\hline 0,0226 & $0,0428 \mathrm{~g} \mathrm{CO}_{2}$.
\end{tabular}

$10 \mathrm{ccm}$ der ursprünglich angewandten Milchsäure verbrauchten vor dem Versuch $8,0 \mathrm{ccm} 0,1-\mathrm{n}-\mathrm{NaOH}$.

$10 \mathrm{ccm}$ der Flüssigkeit in a (Wasser) verbrauchten nach dem Versuch 2,4 ccm 0,1-n-NaOH.

$10 \mathrm{ccm}$ der Flüssigkeit in b (Milchsäure) verbrauchten nach dem Versuch 6,2 ccm 0,1-n-NaOH.

Nach dem Überimpfen auf Bierwürze zeigte es sich, daß 
Über das Verhalten einiger Pilze zu organischen Säuren. I. 289 die Pilze trotz der langen Behandlung mit flüssiger Luft nicht getötet waren.

Im übrigen sind die Ergebnisse dieselben, wie bei Anwendung von getöteten Pilzen. Die Kohlensäureproduktion ist größer in saurer als in wässeriger Lösung, die Säure verschwindet bei Gegenwart der Pilze.

Die Versuche zeigen also ein Verhalten, das dem von Penicillium glaucum gänzlich entspricht. Einer eingehenden Untersuchung steht nur hinderlich im Wege, da $B$ es nicht ganz leicht ist, in genügenden Mengen Pilzmycel zu gewinnen: Noch unbequemer sind aus diesem Grunde Versuche mit Monilia candida anzustellen. Immerhin sei es erwähnt, daß sich dieser Pilz wenigstens gegenüber Ameisensäure dem ebengenannten durchaus vergleichbar verhält. 